\title{
Expert Assessment of Risks Posed by Climate Change and Anthropogenic Activities to Ecosystem Services in the Deep North Atlantic
}

\author{
Claire W. Armstrong ${ }^{1 *}$, Godwin K. Vondolia ${ }^{1,2}$, Naomi S. Foley ${ }^{3}$, Lea-Anne Henry ${ }^{4}$, \\ Katherine Needham ${ }^{5}$ and Adriana Ressurreição ${ }^{6,7,8}$
}

${ }^{1}$ Norwegian College of Fishery Science, UiT - The Arctic University of Norway, Tromsø, Norway, ${ }^{2}$ Norwegian Institute for Water Research, Oslo, Norway, ${ }^{3}$ Socio-Economic Marine Research Unit - SEMRU, National University of Ireland Galway, Galway, Ireland, ${ }^{4}$ School of GeoSciences, University of Edinburgh, Edinburgh, United Kingdom, ${ }^{5}$ Institute of Biodiversity Animal Health and Comparative Medicine, University of Glasgow, Glasgow, United Kingdom, ${ }^{6}$ MARE - Marine and Environmental Sciences Centre, Horta, Portugal, ${ }^{7}$ OKEANOS Centre, University of the Azores, Horta, Portugal, ${ }^{8}$ CCMAR Centre of Marine Sciences, Faro, Portugal

OPEN ACCESS

Edited by:

Daniela Zeppilli, Institut Français de Recherche pour l'Exploitation de la Mer (IFREMER),

France

Reviewed by:

Angelo Fraga Bernardino, Federal University of Espirito Santo,

Brazil

Pedro A. Ribeiro,

University of Bergen, Norway

${ }^{*}$ Correspondence:

Claire W. Armstrong claire.armstrong@uit.no

Specialty section: This article was submitted to Deep-Sea Environments and Ecology, a section of the journal Frontiers in Marine Science

Received: 15 November 2018

Accepted: 12 March 2019

Published: 24 April 2019

Citation:

Armstrong CW, Vondolia GK, Foley NS, Henry L-A, Needham K and

Ressurreição A (2019) Expert

Assessment of Risks Posed by

Climate Change and Anthropogenic Activities to Ecosystem Services

in the Deep North Atlantic.

Front. Mar. Sci. 6:158.

doi: 10.3389/fmars.2019.00158
Sustainable development of the ocean is a central policy objective in Europe through the Blue Growth Strategy and globally through parties to the Convention on Biological Diversity. Achieving sustainable exploitation of deep sea resources is challenged due to the huge uncertainty around the many risks posed by human activities on these remote ecosystems and the goods and services they provide. We used a Delphi approach, an iterative expert-based survey process, to assess risks to ecosystem services in the North Atlantic Ocean from climate change (water temperature and ocean acidification), the blue economy (fishing, pollution, oil and gas activities, deep seabed mining, maritime and coastal tourism and blue biotechnology), and their cumulative effects. Ecosystem services from the deep sea, identified through the Millennium Ecosystem Assessment framework, were presented in an expert survey to assess the impacts of human drivers on these services. The results from this initial survey were analyzed and then presented in a second survey. The final results, based on 55 expert responses, indicated that pollution and temperature change each pose a high risk to more than $28 \%$ of deepsea ecosystem services, whilst ocean acidification, and fisheries both pose a high risk to more than $19 \%$ of the deep-sea ecosystem services. Services considered to be most at risk of being impacted by anthropogenic activities were biodiversity and habitat as supporting services, biodiversity as a cultural service, and fish and shellfish as provisioning services. Tourism and blue biotechnology were not seen to cause serious risk to any of the ecosystem services. The negative impacts from temperature change, ocean acidification, fishing, pollution, and oil and gas activities were deemed to be largely more probable than their positive impacts. These results expand our knowledge of how a broad set of deep-sea ecosystem services are impacted by human activities. Furthermore, the study provides input in relation to future priorities regarding research in the Atlantic deep sea.

Keywords: ecosystem services, climate change, anthropogenic impacts, risk, deep sea, North Atlantic Ocean, blue growth 


\section{INTRODUCTION}

Although our oceans and seas are "out of sight, out of mind" according to much of society, we are becoming increasingly aware of the fact that marine ecosystems are highly impacted by climate change and our endeavors to exploit living and nonliving marine resources (Halpern et al., 2008). Despite their remoteness and difficulty to access, the demise of the deep ocean is particularly noteworthy as it is the largest but least known biome on Earth. The deep North Atlantic Ocean has been studied for the last two centuries, and is now known to harbor ecosystems that support a biologically rich variety of life that perform key functions to global biogeochemical cycles, e.g., cycling of primary production, carbon and nutrients from the ocean surface to the deep seafloor (Oevelen et al., 2009; Vanreusel et al., 2010; Beazley et al., 2013). Complex three-dimensional ecosystems formed by cold-water corals, sponges, and topographically complex seamounts and hydrothermal vents not only support high and sometimes unique or endemic species, but these ecosystems also provide many ecosystem goods and services, which contribute to maritime economic activities that underpin the socio-economic wellbeing of Atlantic nations and their citizens (Galparsoro et al., 2014). These services include nutrient cycling and the biological pump, waste absorption and detoxification, fisheries and other deep ocean industries, bioprospecting and a number of cultural services related to education and science, aesthetic and inspirational contributions (Thurber et al., 2014). There is a lack of environmental baselines and assessments in relation to human interactions with the deep sea, but research points to three main impact contributors; the earlier days' disposal and dumping, current resource exploitation, and future climate change, impacting on natural resources and different habitat types, as described in some detail by Ramirez-Llodra et al. (2011). Deep sea habitats and services have undoubtedly been degraded by disposal and resource exploitation, and are now further challenged by unprecedented rates of climate change that will see the deep North Atlantic experience reduced oxygen levels and food supply to the seafloor, lower $\mathrm{pH}$ and a rise in deep ocean temperatures (Sweetman et al., 2017).

In addition to climate change and historic types of resource exploitation dating back to antiquity (fisheries) and into the last century (oil and gas), the European Commission Blue Growth Strategy seeks to support sustainable growth in the North Atlantic across five sectors: aquaculture, maritime and coastal tourism, blue biotechnology, ocean energy and seabed mining (EC, 2012). This strategy may pose a challenge to the business and policy objectives, seeking to balance societal needs with environmental sustainability.

One way to consider the balance between the blue growth economic agenda and sustainability is to assess the potential impacts or risks posed by different forms of human activity on the ecosystem services provided by the deep sea. It may also be assumed that an assessment of impacts and risks will inform marine spatial planning (MSP). MSP calls for due regard in relation to various pressures from human activities and climate change on marine ecosystems, their services and economic development (Ntona and Morgera, 2018). These impacts affect and pose risks in relation to services that the ecosystems provide to humans. To assess any form of risk, consequences and probability of hazard occurrences need to be identified. There is a multitude of studies assessing risks of specific activities, such as oil spills, aquaculture or shipping on specific resources, environments, ecosystems or their functions in marine ecosystems (Soares and Teixeira, 2001; Olita et al., 2012; Copp et al., 2016). However, there are few studies that integrate risk assessments and ecosystems services [see Nienstedt et al. (2012) for a terrestrial example], or at least these are mainly limited to discussion regarding the approach (Faber and van Wensem, 2012; Galic et al., 2012). There are several reasons for this gap. For one, the assessment of risks in relation to natural environments or ecosystems is often very demanding in itself. Knowledge is limited (particularly so in the deep sea), and the consequences can be highly diverse as well as controversial. Bringing the risk analysis one step further, to ecosystem services, can, therefore, be even more challenging. A second issue is; who are the experts that should assess the risk to ecosystem services? Although the scientific knowledge can provide expert input regarding risks to ecosystems, it is not clear which expert body can provide expertise in relation to ecosystem services, i.e., services from ecosystems that provide benefits to humans (MEA, 2005). Many economists assess values connected to ecosystem services (TEEB, 2010), but are they not necessarily the experts to assess unvalued risks, i.e., risks to ecosystem service provision as such? Though social scientists, in general, have not criticized the concept of ecosystem services to the same degree that others from different disciplines have (Morelli and Møller, 2015; Silvertown, 2015), they cannot be said to completely embrace it (Sullivan, 2010). Furthermore, social science in this domain has more often focused on the interaction between humans in nature, power structures and knowledge acquisition, rather than individual or societal beneficiary interactions with ecosystems. This is changing, however, with input from social sciences and the humanities increasingly finding its place, for instance in the intergovernmental science-policy panel on biodiversity and ecosystem services (IPBES) (Stenseke and Larigauderie, 2018). Nonetheless, our study links ecosystems and services via the risk aspect, making it acceptable to survey largely natural scientists.

Why is it of interest to assess risks to ecosystem services, rather than environments, ecosystems or ecosystem functions? Clearly, the drive within the EU for marine ecosystem-based management is central in the aim for a broader perspective on the use of marine resources (MSFD, 2008). The concept of ecosystem services, which has in recent years increasingly appeared in research, but also in policy and management (see for instance the MAES: Mapping and Assessment of Ecosystems and their services, under Action 5 of the EU Biodiversity Strategy to 2020, and the EU Blue Growth Strategy) brings nature's contributions to humans to the forefront. Assessing risks to these services brings the consequences of human drivers directly in contact with societal aspects, i.e., the risks are brought closer to the issues that managers and politicians are directly considering. Although risks to ecosystems and their functions are of course important, there is at least one layer of knowledge between the output of these kinds of assessments and the human dimensions that managers 
and policy makers relate to. In going directly to the ecosystem services, we bring the risks of human drivers closer to home. Though there is mounting scientific evidence surrounding risks to ecosystems, the many linkages between ecosystems and the multitude of ecosystem services they provide are not always well known or clearly identified, especially in the deep sea. Identifying risks to ecosystem services that deep sea ecosystems supply illustrates more clearly the potential losses that society may experience if the human drivers of change in these environments are not taken into account. In addition, the deep sea is often both spatially and temporally distant to the services that humans value, and therefore, all the more important to identify the riskiest drivers, and from this provide input into where more work is required to mitigate or adapt to the risks involved.

In this paper, we use a Delphi survey approach to assess risks to deep sea ecosystem services within the North Atlantic. This is in contrast to previous work which has considered effects on ecosystems and their functions (Ahnert and Borowski, 2000), effects by single drivers (Gornitz et al., 1994) and the effects on ecosystem services in coastal areas (Hayes and Landis, 2004), which has most often been the focus in the literature. The Delphi approach is an iterative expert-based survey approach, in order to see whether perceptions may reach greater consensus based on information about the choices of peers in a previous round of the survey. Due to the lack of specific experts in this matter, we have chosen to use a broad set of expertise to assess the risks to ecosystem services in the deep sea. Our Delphi study respondents are members of two EU Horizon 2020 projects, consisting of a large variety of mostly biological and oceanographic expertise in relation to the North Atlantic deep-sea. The experts span physical oceanography, ecosystem modeling, deep sea ecology and genetics, natural resource economics and social science, as well as marine policy. We assess risks of human activities or drivers on ecosystem services in the deep sea, using expert elicited risk assessments in a Delphi format. The results expand our knowledge of how a broad set of ecosystem services from the deep-sea are impacted by human activities. Furthermore, the study provides input in relation to future priorities, regarding research in the North Atlantic. The remainder of the paper follows this structure: The methodologies are discussed in the next section. The results are presented in section "Results", with discussions and conclusions in section "Discussions".

\section{MATERIALS AND METHODS}

We used a Delphi survey to assess the risks of human drivers to deep-sea ecosystem services. The Delphi method has its origins from the RAND Corporation in the 1950s and 1960s and was largely motivated by the need for improved forecasting and securing some form of judgment convergence (Dalkey, 1968). Over the years it has been utilized in a multitude of different assessments spanning from health issues (Steen et al., 2014; Keller et al., 2015) to challenges in the pulp and paper industry (Toppinen et al., 2017). The method is largely used to obtain some form of opinion consensus, whilst avoiding the influence of dominant individuals. In recent years, Delphi surveys have increasingly been applied to environmental issues, such as valuation (Strand et al., 2017), and especially in relation to issues where there is limited knowledge, either in relation to the ecology (Filyushkina et al., 2018) or the values (Scolozzi et al., 2012; Filyushkina et al., 2018).

The Delphi method relies on a panel of experts to gather information; on a subject with limited knowledge. This expert opinion is gathered through an iterative, anonymous survey with feedback. The survey is sent around twice or more, and information regarding the results of the previous round is distributed to allow the experts to evaluate their assessment and to see if there may be some agreement or convergence regarding the issue surveyed. The objective is to allow information produced by an expert group to be evaluated, building consensus over time (see the stages in the Delphi approach in Table 1).

Though the Delphi process is considered to be more reliable than a single survey, the method has been critiqued for group pressure, rather than knowledge development, leading to consensus in repeated surveys (Woudenberg, 1991). However, the Delphi approach is also roundly defended, especially in relation to complex issues (de Loë et al., 2016) and topics where information is not easy to come by Landeta (2006). Also, in other fields where surveys are used, giving respondents time to reflect, discuss and gather information is seen as a way to secure responses that are more reliable (MacMillan et al., 2006).

\section{Risk Assessment Survey}

The risk assessment survey was developed based on literature on ecosystem services in the deep sea (Armstrong et al., 2012; Galparsoro et al., 2014; Thurber et al., 2014), and determination of relevant human drivers within the research group (see Table 2).

\section{Ecosystems Services}

Ecosystem services are described as those services that ecosystems provide for human wellbeing. There exists a number of different ecosystem service frameworks that have been developed over the last 15 years. We apply the Millennium Ecosystem Assessment's (MEA, 2005) framework in our analysis (Figure 1). This framework includes supporting services that feed into the direct services to humans; the provisioning, regulating and cultural services. A number of newer frameworks,

TABLE 1 | Stages in the Delphi survey approach.

\begin{tabular}{ll}
\hline Steps & \\
\hline 1 & Definition of problem \\
2 & Selection of experts \\
3 & Survey instrument development \\
4 & Testing of survey instrument \\
5 & Distribute 1st survey \\
6 & Analysis of 1st round results, and development of \\
7 & presentation for 2nd survey \\
8 & Distribute 2nd survey \\
& Analysis of 2nd round results, comparison to 1st round, \\
& develop report
\end{tabular}


such as TEEB (The Economics of Ecosystems and Biodiversity), CICES (The Common International Classification of Ecosystem Services) and IPBES, do not include supporting services explicitly in their service portfolio (TEEB, 2010; CICES, 2013; IPBES, 2017). The motivation for not including the supporting services is largely due to the issue of double counting values. When monetarily estimating the value of ecosystem services, supporting services cannot be valued separately, as their values are inherently included in the value of the direct services that they feed into. Since we do not undertake valuation in this study, double counting is not an issue we need to consider. Furthermore, in our study area (the deep sea), most ecosystem services are removed in time and space from humans, hence very many services are of the supporting type (Armstrong et al., 2012).

\section{Human Drivers}

The human drivers of risk to ecosystems and their services were identified through discussions with experts and include some of the key areas identified for development within the EU Blue Growth Strategy (Table 2) (aquaculture was not considered a risk to the study area).

\section{Defining Risk}

Risk is the product of two entities, consisting of (1) some measure of the consequences of an occurrence and (2) the likelihood
TABLE 2 | Human drivers identified for the Delphi survey risk assessment.

\section{Identified Human Drivers}

Temperature Change

Ocean Acidification

Fishing

Pollution

Oil and Gas

Mining

Tourism

Blue Biotechnology

that the occurrence will take place. Usually, the occurrence is defined as some hazard. However, occurrences need not be hazards causing negative effects, though this is usually what we worry most about and are most interested in identifying. In our case, the hazards are presented as a number of different human drivers or their combination, impacting on ecosystem services. These drivers need not always lead to negative effects on all ecosystem services and in some cases provide positive effects, or there may indeed be reasons to believe some drivers may have both positive and negative effects. Our study involves a large number of ecosystem services and human drivers across the North Atlantic Ocean. It is recognized that there is currently limited knowledge of the deep sea and this leads to increased uncertainty in the study. As such, experts could note both positive

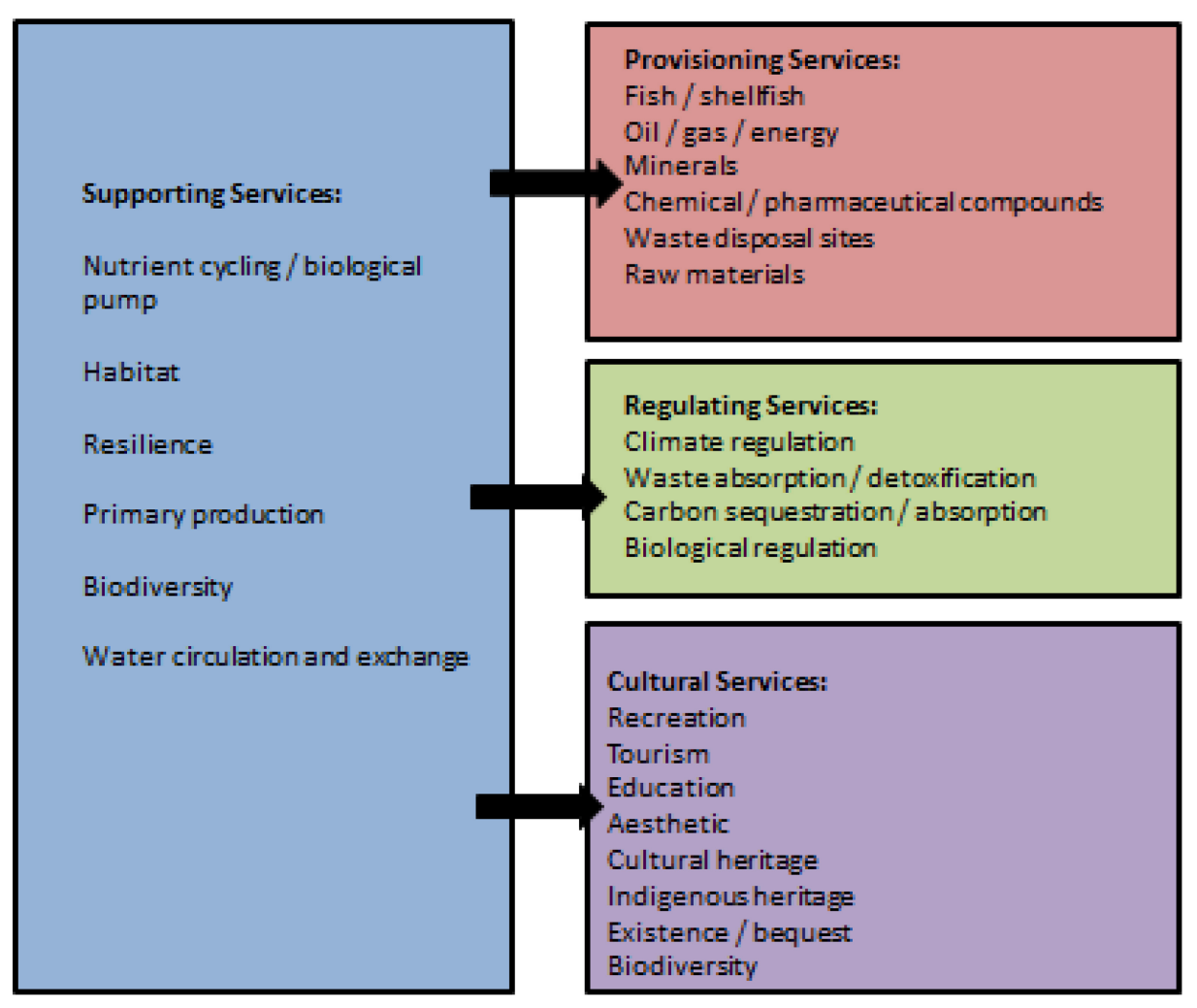

FIGURE 1 | Ecosystem services in the deep sea, using the Millennium Ecosystem Assessment framework. 
and negative effects in our assessment. Hence, the assessment allows for positive and negative effects, with a scale of 1-5 (from very low severity to very high severity), or alternatively neither being applicable for some drivers in relation to some ecosystem services. The likelihood of the effect occurring is also measured on a scale of 1-5 (very low probability to very high probability). Ideally, we should have included probabilities and specified severities. However, in order to keep the survey as brief as possible, we kept to these simplifying descriptions. Such a presentation of risk may, however, be problematic, and must be used with caution (Cox, 2008). However, we do not estimate a risk measure, but rather present the graphical combination of likelihood and severity. Risk assessment, in general, can also be critiqued based on normative aspects and in relation to problems of aggregation (Stirling, 1998). However, caution is largely suggested in relation to decision-making in high-risk situations. For the use of assessing risk aspects in relation to broad categories of ecosystem services, such as we are carrying out here, many of the cautions are less problematic.

It is, however, worth noting the choice of grid lines in the risk matrix (i.e., where the high, moderate and low risk is assigned) is highly subjective. Clearly, these lines should be determined by some aim to "minimize the maximum loss of misclassified risks" (Cox, 2008, p 510), but this requires a lot more knowledge regarding consequences than is available for our study and is seldom problematized in risk assessments.

\section{The Survey}

An initial survey was developed and pre-tested. Following revisions to the survey, the first round of the Delphi ecosystem service risk assessment was held at the 2nd EU Horizon 2020 project ATLAS' General Assembly in April 2017. The session included a brief introduction to the aims of the work, the Delphi method and ecosystem services. The participants were given explanatory material (see Appendix 1) and the survey in an Excel sheet via email (see Appendix 2). Anonymity was guaranteed, as no data was collected that could identify the respondents. All respondents could pull out of the survey at will. While some surveys were returned during the project meeting, the majority were submitted in the following weeks. A total of 30 surveys were submitted and included for analysis. The responses to the first round survey were analyzed; figures were produced to present relevant results for the next round and a new survey using results from the first survey was developed using SurveyMonkey ${ }^{1}$ (see Appendices $\mathbf{3}$ and $\mathbf{4}$ for the second survey and an example of the online survey). In our presentation of the results from the first survey, we show first the perceptions of negative effects, as these are of most interest in relation to policy, research, mitigation and adaptation. We presented the risk reporting matrices in the fashion of likelihood and effect as shown in Figure 2, where the two axes are represented by rank numbers. This representation is not uncommon (see for instance FAO guidelines for Ecosystem Approach to Fisheries ${ }^{2}$.

\footnotetext{
${ }^{1}$ https://www.surveymonkey.net/

${ }^{2}$ http://www.fao.org/fishery/eaf-net/eaftool/eaf_tool_4/en\#EAFToolEAFToolSynergy
}

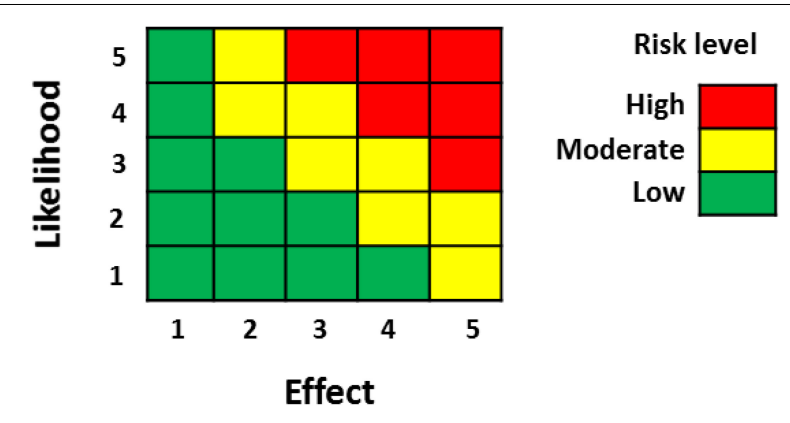

FIGURE 2 | Risk reporting matrix.

The second survey was distributed, by e-mail, to the ATLAS project members at the end of October 2017, and two reminders were sent out. By mid-November, 20 surveys from participants who had taken part in the first round were received. In April 2018, a special session was held for all project members at the EU Horizon 2020 project SponGES' General Assembly. The project members were asked to complete the second version of the Delphi ecosystem service risk assessment online, using SurveyMonkey. Prior to completing the survey, respondents received a brief presentation about the Delphi method, ecosystem services and structure of the survey, including the results from the first survey. These initiatives resulted in a total of 55 responses. The results from the ATLAS second round and the SponGES assessment were pooled and analyzed, and compared to the first round, as presented in the Results section below.

\section{RESULTS}

The results presented focus on the second round of the Delphi survey. The first round was employed as an initial information gathering exercise, which provided information for use in the second round. Results from the first round of the Delphi survey are available in Appendix 3 and equivalent results from the second round are presented in Appendix 5. We focus on the results from the second survey, which is reasonable given a Delphi approach, where the second survey includes more assessment by the respondents. In some cases, the results may appear different in the two surveys, but we find hardly any statistically significant difference, and hence only present the figures from the second survey in this section (see the Appendices for results from the first survey).

In the following we present the results from two forms of risk; (1) the risk resulting from anthropogenic activities (human drivers) on each ecosystem service identified to the deep sea and (2) the perceived level of risk associated with each human driver. As discussed previously, both positive and negative effects were surveyed, but in this section, we focus on the negative effects.

Fifty-five (55) responses were received from experts participating in the ATLAS and SponGES projects to the second survey, out of which $47 \%$ (all from the ATLAS project) participated in the first round. For each ecosystem service, the number of human drivers that pose high, medium and low risks 
are identified. The median severity and the median likelihood of the negative effects are presented for all services and drivers, as the data gathered is at the ordinal level. The high, medium and low risks connected to the negative effects of human drivers on different ecosystem services are presented in Figure 3. It is shown that habitat and biodiversity, both supporting ecosystem services, are at high risk from six out of nine human drivers. Biodiversity, when it is considered a cultural ecosystem service, is at high risk from five human drivers. The provisioning ecosystem service of fish/shellfish is at high risk from four of the human drivers. Resilience is at high risk from two human drivers while the remaining ecosystem services are at high risk from at most one human driver, if any at all.

The ecosystem services at low risk from a high number of human drivers are waste disposal and raw materials (both provisioning ecosystem services) and a supporting ecosystem service of primary production. None of these three ecosystem services are at high risk from human drivers.

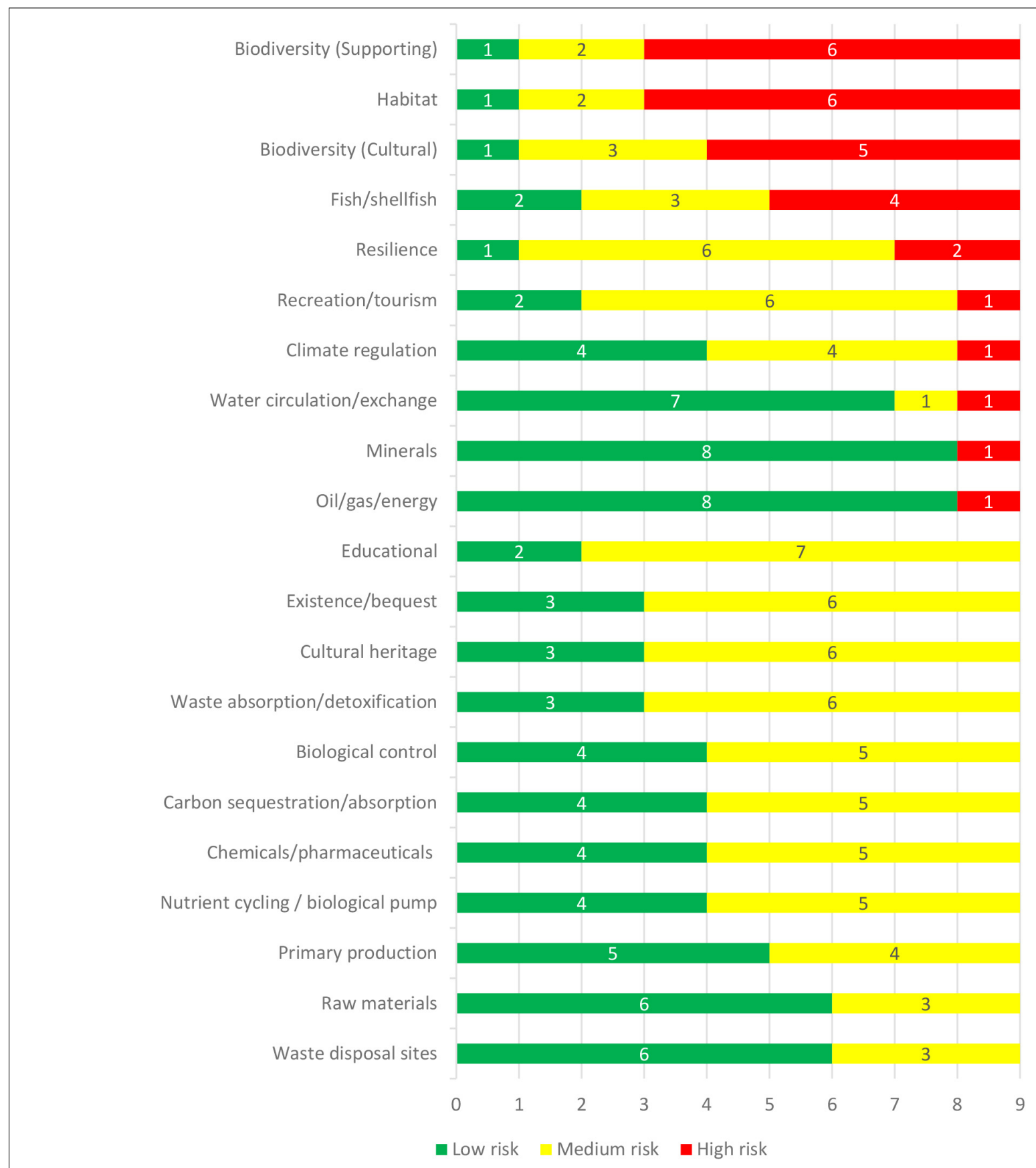

FIGURE 3 | Ecosystem service risk levels from the negative effects of different human drivers. The $x$-axis represents the number of drivers within each risk category. 


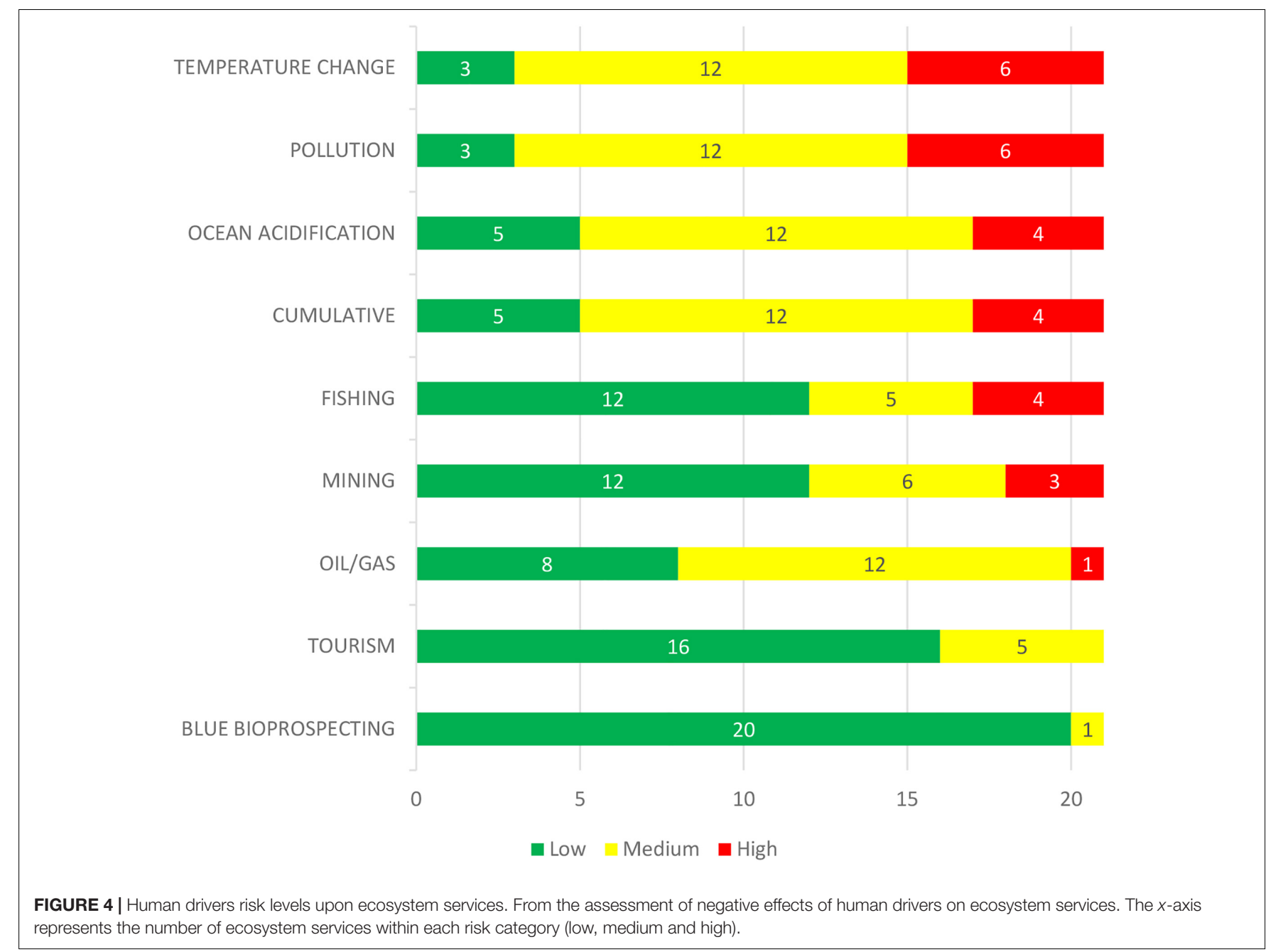

The second objective was to assess the level of risk associated with the human drivers specifically. In Figure 4, we illustrate the different human drivers and how they impact on the 21 ecosystem services as regards to high, medium and low risks. This is used to identify the anthropogenic activities (human drivers) that are perceived to have the highest level of risk to ecosystem services.

In Figure 4, pollution and temperature change both cause high risk to five ecosystem services. This means that pollution and temperature pose high risk to $28 \%$ each for the ecosystem services identified. Ocean acidification, fishing and the cumulative impacts cause high risk to four ecosystem services each. This corresponds to about $19 \%$ of the identified ecosystem services. These are followed by mining, causing high risk to three ecosystem services. Tourism and blue biotechnology are not perceived to have any high risk impacts on ecosystem services, and oil/gas activities are only perceived to be high risk in relation to oil/gas/energy as provisioning services.

The rankings of human drivers remain the same in both the first and second surveys, except for temperature change which replaces ocean acidification as the greatest risk in the second survey. In addition, the risks of the six high risk human drivers increase. For instance, in the first round, pollution posed high risk to four ecosystem services and this increases to six in the second survey. Adverse impacts of tourism remain the same, but risk perceptions of blue biotechnology declined in the second survey. There is, however, no statistical difference between the average number of ecosystem services at high, medium and low risk in the first and second surveys as can be seen in Table 3. We also observe that the variation only declined in the second round for the medium risk case. This may be due to the difference in the number of participants who responded to the two rounds. It is worth noting that there is large variation in the high-risk ecosystem services, as well as for the human drivers.

In Figure 5, the positive effects of the different human drivers on different ecosystem services are presented, in order to compare the expectation of positive versus negative effects of different human drivers. We chose not to develop a single risk measure (for instance by using the product of the two digits from effect and likelihood), despite this not being uncommon in the literature (Staples et al., 2014), as products of ranked measures may give spurious and therefore unreliable results when compared (Cox, 2008; Hubbard and Evans, 2010). Each separate figure shows the positive and negative effects, using green and red colored bubbles, respectively. The size of the 
TABLE 3 | Mean number of human drivers posing high, medium and low risk to ecosystem services, and mean number of ecosystem services at high, medium and low risk from human drivers, in the first and second survey.

\begin{tabular}{|c|c|c|c|c|c|c|}
\hline & \multicolumn{3}{|c|}{ Human drivers } & \multicolumn{3}{|c|}{ Ecosystem services } \\
\hline & First survey & Second survey & $\begin{array}{c}T \text {-values of mean } \\
\text { differ-ence test }\end{array}$ & First survey & Second survey & $\begin{array}{c}T \text {-values of mean } \\
\text { difference test }\end{array}$ \\
\hline High risk & 0.81 (1.03) & 1.33 (2.06) & -1.04 & 2.00 (1.73) & $3.11(2.31)$ & -1.15 \\
\hline Medium risk & $3.90(2.07)$ & $4.57(1.75)$ & 1.13 & $10.44(4.42)$ & $8.55(4.30)$ & 0.92 \\
\hline Low risk & 3.52 (1.57) & 3.72 (2.23) & -0.40 & 8.33 (6.08) & $9.33(6.04)$ & -0.35 \\
\hline
\end{tabular}

Numbers in parenthesis are standard deviations. T-values of mean difference test between the two surveys.

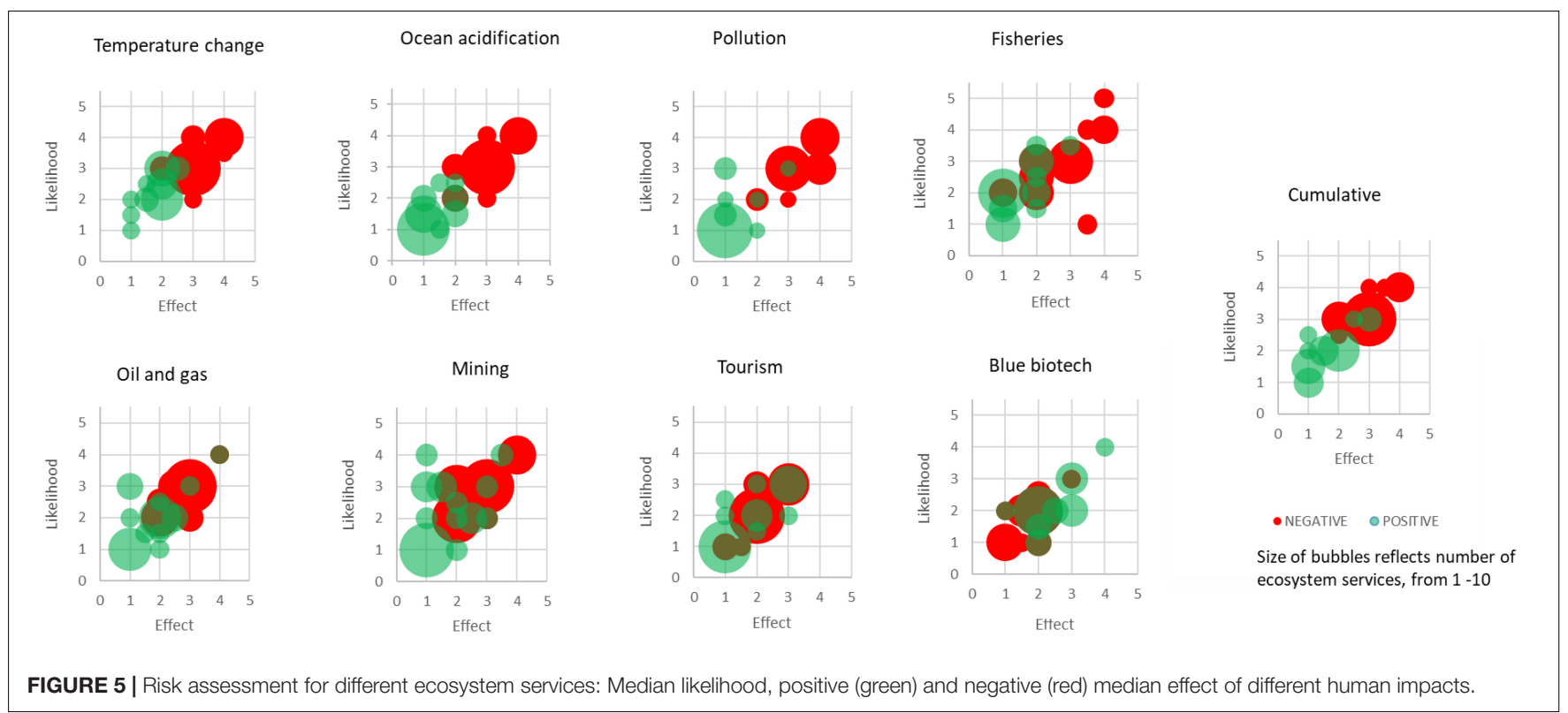

bubble illustrates how many services are represented at each point of likelihood and severity of effect. Here we observe that for temperature change, ocean acidification, pollution, fisheries, oil/gas activities and cumulative effects, the negative effects come at far higher risk levels than the positive. This can be seen from the red bubbles concentrating to the upper right of the figures, while the green are more to the left. For mining and tourism, this effect is less clear. We can observe that for blue biotechnology, the positive effects come at far higher risk levels than the negative.

\section{Comparison of the First and Second Survey, and Respondent Certainty}

Comparing the first and second surveys, risk perceptions regarding human drivers give some appearance of worsening in the second survey relative to the first (for graphical comparison, see Figure 3 above for the second survey and Figure A4 in Appendix 5 for the first survey). The number of human drivers that pose high risk increases from three to six for biodiversity (as a supporting service), two to six for habitat, three to five for biodiversity (as a cultural service) and two to four for fish/shellfish. However, using simple $t$-tests we find no statistically significant difference between the average of number of human drivers classified as high, medium and low risk to deepsea ecosystem services, identified in the first and second surveys, as can be seen in Table 3. We also observe that the variation only declined in the second round for the medium risk case. This may be due to the difference in participants in the two rounds. It is worth noting the large variation in the high-risk human drivers.

We also assessed whether perceptions differed from the first survey to the second, regarding the average number of human drivers posing high risk to ecosystem services, for those services that are perceived to be at high risk from at least one human driver, as shown in Table 4. Using $t$-tests, we reject the

TABLE 4 | Conditional mean number of human drivers posing high risk to ecosystem services, for those services that are perceived to be at high risk from at least one human driver.

\begin{tabular}{lllc}
\hline & First survey & Second survey & $\begin{array}{c}\boldsymbol{T} \text {-values of mean } \\
\text { difference test }\end{array}$ \\
\hline High risk & $1.7(0.8232726)$ & $2.8(2.20101)$ & -1.4803 \\
Medium risk & $3.6(1.074968)$ & $2.7(2.162817)$ & 1.1784 \\
Low risk & $3.6(1.429841)$ & $3.5(3.02765)$ & 0.0944 \\
\hline
\end{tabular}

Numbers in parenthesis are standard deviations. T-values of mean difference test between the two surveys. 
null hypothesis that the two conditional means are different, at the $5 \%$ level.

Although the rankings of ecosystem services in terms of high risk remain almost the same in both the first and second survey, there were some changes in rankings of ecosystem services that are low risk. Risk perceptions of human drivers to educational services increased in the second survey but waste absorption, carbon sequestration, chemicals/pharmaceuticals and nutrient cycling were ranked lower in the second survey than in the first survey. However, the ecosystem services' risk levels show similarly small changes as observed for the human drivers (see Table 3).

After the risk assessment, we asked about respondents' certainty in their responses using a scale from 1 to 5 (with 1 being very uncertain and 5 being very certain). The median level of the certainty score is 3 for both surveys, but the average certainty increased slightly from 2.56 in the first survey to 2.82 in the second round. A $t$-test found no statistical difference (at $5 \%$ level) between the expressed certainty in the two surveys.

We also asked the respondents to identify which ecosystem services that they are certain about. The main ecosystem services identified here include biodiversity, climate regulation and habitat. The cascading effects of these on other ecosystem services, especially under the supporting and regulating ecosystem services, were also noted. Instead of ecosystem services, some of the respondents indicate the impacts of human drivers that they are certain about. The human drivers cited include temperature change, ocean acidification, mining, pollution, tourism, biotechnology and fishing.

We also offered the respondents an opportunity to identify which ecosystem services they were most certain about. Although not all respondents responded to this query, the main ecosystem services identified included biodiversity, climate regulation and habitat. Some of the ecosystem services that respondents were uncertain about, regarding how they will be impacted by human drivers, include carbon cycle, oceanographic and water circulation, in addition to the combined effects of the undergoing changes in ecological functions on regulating services. Some of the respondents mention issues, such as the ecosystem services framework, the political will to take actions that are required to address the effects of human drivers and the scoring and monetary valuation of these effects.

During the survey, we asked the experts to give opinions on the validity and usefulness of the ecosystem services framework for understanding human dependence on the marine environments. The general impressions from these responses indicate that the majority of experts think that the ecosystem services framework is very useful. However, they also believe that it can be improved, especially strengthening the scientific basis of the framework. Some reservations are expressed about the intangible nature of some of the ecosystem services, such as cultural and supporting services, as well as the fact that ecosystem service frameworks can mask the importance of natural processes and functions that underpin the framework itself. Some propose that other frameworks should be developed to provide alternative perspectives.

\section{DISCUSSION}

The survey points to four perceived high risk human drivers: pollution, temperature change, ocean acidification and fisheries, in addition to the cumulative effects. These results are similar to the findings of Halpern et al. (2008), who, using a number of databases combined with an expert judgment based area assessment, showed how Northern Atlantic ecosystems, especially in the east, are highly impacted. The authors show that the climate drivers (sea temperature, UV and ocean acidification), impact the largest ocean areas. However, though fishing covers far less area, different aspects of fishing (different types of by-catch as well as habitat modification), were perceived to pose similar threat levels as that of the climate factors. Pollution was given far less attention in the Halpern et al. (2008) study than it has been in our results. This may be due to further knowledge about the extent of marine pollution over the last 10 years, or because pollution is perceived to have a greater impact on ecosystem services than on marine ecology, the latter which was the focus of the Halpern et al. (2008) study. Interstingly, in our study, oil/gas and mining are considered to be far less risky in relation to ecosystem services than the four main high risk drivers. Blue biotechnology and tourism are perceived to provide the greatest positive effects and likelihoods, with oil/gas and mining following them. In conclusion, newer blue growth industries do not seem to involve the greatest risk to ecosystem services. Indeed, it seems to be the larger global problems of climate, pollution and fisheries that are perceived to pose the highest risks to marine ecosystem services.

The main contribution of this study is to focus on risk to ecosystem services, rather than marine ecology or ecosystems, which is what are usually studied. Here we observe that the ecosystem services perceived to be most threatened, i.e., services with high risk levels in relation to most human drivers, are fish and shellfish, biodiversity (both as a supporting and a cultural service) and habitats. Provisioning (fish/shellfish), cultural (biodiversity) and supporting services (biodiversity and habitats) are therefore believed to be at risk from the largest number of human drivers. The only regulating service understood to be at risk was climate regulation, due to temperature change. Indeed, supporting services were perceived to be the most at risk. This is noteworthy, as when focusing on ecosystem services, most of the newer frameworks (TEEB, 2010; CICES, 2013; IPBES, 2017) largely do not include supporting ecosystem services, but rather focus on the final provisioning, regulation, and cultural services. An important message is that if the focus is only given to the three ecosystem service types that directly impact humans, we may clearly ignore important impacts and their risks. This is particularly meaningful in the deep sea.

This study has several qualifications worth mentioning. One is that the numbers of responses are limited and having more respondents in the first version would have been advantageous. Furthermore, organizing the likelihood in a different fashion, for instance in probabilities rather than ranks, would allow a more multiplicative presentation (probability multiplied by effect), though as mentioned earlier, this is not without its problems. Giving the respondents more information would be good but 
must be evaluated against the time needed to carry out the survey. Yet more information in the second survey, regarding the variance in the results, could have been informative. The survey is very large and demands a lot of the respondents. One option could be to limit a followup survey to the most high risk drivers and ecosystem services, in order to probe these further. Though a qualitative assessment of respondent uncertainty was included, a more systematic uncertainty assessment related to risks would benefit a future study, providing extra input on knowledge gaps and further research needs.

The study underlines a number of issues. For one, there seems to be large degree of uncertainty around the level of high risk that different human drivers pose. Though pollution, temperature change, ocean acidification and fisheries, and the cumulative effects are, on average, deemed to pose high risk to several ecosystem services, there is substantial variation in this perception. This points to a need for further study in relation to these drivers and their linkages to ecosystem services. There is also considerable variation amongst the respondents, regarding the ecosystem services perceived to be at high risk. The fact that it is the high-risk drivers and services that show the greatest variation is concerning, and stresses the need for more research into the pressures and their responses, especially in the deep sea. Furthermore, the focus on supporting services being at risk is notable. The fact that most of the respondents were natural scientists may be one explanation for this result, and points to the need for greater inclusion of other disciplinary fields in surveys that involve ecosystem services. However, it should not be surprising that supporting services are central services in the deep sea, and that their importance may well surpass many other services that receive more attention in shallower and coastal waters.

Achieving sustainable exploitation of deep sea resources is challenged by the huge uncertainty around the many risks posed by human activities on these remote ecosystems and the goods and services they provide. This study contributes to the blue growth development and MSP in the deep sea by identifying human activities and climate change effects that may have an impacts on ecosystem services. The identification of the levels of risk associated with different human activities,

\section{REFERENCES}

Ahnert, A., and Borowski, C. (2000). Environmental risk assessment of anthropogenic activity in the deep-sea. J. Aqu. Ecosyst. Stress Recov. 7, 299-315. doi: 10.1023/A:1009963912171

Armstrong, C. W., Foley, N., Tinch, R., and Van Den Hove, S. (2012). Services from the deep: steps towards valuationof deep sea goods and services. Ecosyst. Serv. 2, 2-13. doi: 10.1016/j.ecoser.2012.07.001

Beazley, L. I., Kenchington, E. L., Murillo, F. J., and Sacau, M. D. M. (2013). Deepsea sponge grounds enhance diversity and abundance of epibenthic megafauna in the Northwest Atlantic. ICES J. Mar. Sci. 70, 1471-1490. doi: 10.1093/icesjms/ fst 124

CICES (2013). Common International Classification of Ecosystem Services Version 5.1. Available at: http://cices.eu (accessed July 6, 2018).

Copp, G. H., Godard, M. J., Russell, I. C., Peeler, E. J., Gherardi, F., Tricarico, E., et al. (2016). A preliminary evaluation of the european non-native species in and the perceived level of risk to ecosystem services will help inform future development and potentially maintain ecosystems in the deep sea.

\section{AUTHOR CONTRIBUTIONS}

GV and CA analyzed the data. CA initiated the writing of the manuscript. All authors were substantially involved in the survey development and data collection, contributed to development of the results used and the final product in significant ways, and provided approval for publication.

\section{ACKNOWLEDGMENTS}

We thank members of the Horizon 2010 ATLAS - A TransAtLantic Assessment and deep-water ecosystem-based Spatial management plan for Europe, and SponGES - Deep-sea Sponge Grounds Ecosystems of the North Atlantic an integrated approach toward their preservation and sustainable exploitation, for their participation in the surveys. We would like to acknowledge the help of Joana Xavier, Hans Tore Rapp, and Detmer Sipkema for facilitating the opportunity of presenting and applying the Delphi survey among the experts of the Horizon 2020 project SPONGES. AR acknowledges Fundação para a Ciência e Tecnologia (FCT), through postdoctoral grant (SFRH/BPD/102494/2014) and the strategic project UID/MAR/04292/2013 granted to MARE. We acknowledge funding from the European Union's Horizon 2020 Research and Innovation Program under grant agreement no. 678760 (ATLAS). This output reflects the authors' views only and the European Union cannot be held responsible for any use that may be made of the information contained therein.

\section{SUPPLEMENTARY MATERIAL}

The Supplementary Material for this article can be found online at: https://www.frontiersin.org/articles/10.3389/fmars. 2019.00158/full\#supplementary-material

aquaculture risk assessment scheme applied to species listed on Annex IV of the EU Alien Species Regulation. Fish. Manage. Ecol. 23, 12-20. doi: 10.1111/ fme. 12076

Cox, L. A. Jr. (2008). What's wrong with risk matrices? Risk Anal. 28, 497-512. doi: 10.1111/j.1539-6924.2008.01030.x

Dalkey, N. C. (1968). Predicting the Future. Santa Monica, CA: The Rand Corporation.

de Loë, R. C., Melnychuk, N., Murray, D., and Plummer, R. (2016). Advancing the state of policy delphi practice: a systematic review evaluating methodological evolution, innovation, and opportunities. Technol. Forecast. Soc. Change 104(Suppl. C), 78-88. doi: 10.1016/j.techfore.2015.12.009

EC (2012). Blue Growth; Opportunites for Marine and Maritime Sustainable Growth. Brussels: European Commission.

Faber, J. H., and van Wensem, J. (2012). Elaborations on the use of the ecosystem services concept for application in ecological risk assessment for soils. Sci. Total Environ. 415(Suppl. C), 3-8. doi: 10.1016/j.scitotenv.2011.05.059 
Filyushkina, A., Strange, N., Löf, M., Ezebilo, E. E., and Boman, M. (2018). Applying the Delphi method to assess impacts of forest management on biodiversity and habitat preservation. For. Ecol. Manage. 409, 179-189. doi: 10.1016/j.foreco.2017.10.022

Galic, N., Schmolke, A., Forbes, V., Baveco, H., and Van Den Brink, P. J. (2012). The role of ecological models in linking ecological risk assessment to ecosystem services in agroecosystems. Sci. Total Environ. 415(Suppl. C), 93-100. doi: 10.1016/j.scitotenv.2011.05.065

Galparsoro, I., Borja, A., and Uyarra, M. C. (2014). Mapping ecosystem services provided by benthic habitats in the European North Atlantic Ocean. Front. Mar. Sci. 1:23. doi: 10.3389/fmars.2014.00023

Gornitz, V. M., Daniels, R. C., White, T. W., and Birdwell, K. R. (1994). The development of a coastal risk assessment database: vulnerability to sea-level rise in the U.S. Southeast. J. Coast. Res. 12, 327-338.

Halpern, B. S., Walbridge, S., Selkoe, K. A., Kappel, C. V., Micheli, F., D'agrosa, C., et al. (2008). A global map of human impact on marine ecosystems. Science 319, 948-952. doi: 10.1126/science. 1149345

Hayes, E. H., and Landis, W. G. (2004). Regional ecological risk assessment of a near shore marine environment: cherry point, WA. Hum. Ecol. Risk Assess. 10, 299-325. doi: 10.1080/10807030490438256

Hubbard, D., and Evans, D. (2010). Problems with scoring methods and ordinal scales in risk assessment. IBM J. Res. Dev. 54, 2:1-2:10. doi: 10.2460/javma.242. 10.1359

IPBES (2017). Update on the Classification of Nature's Contributions to People by the Intergovernmental Science-Policy Platform on Biodiversity and Ecosystem Services. Bonn: Germany.

Keller, H. H., Mccullough, J., Davidson, B., Vesnaver, E., Laporte, M., Gramlich, L., et al. (2015). The Integrated Nutrition Pathway for Acute Care (INPAC): building consensus with a modified Delphi. Nutr. J. 14:63. doi: 10.1186/s12937015-0051-y

Landeta, J. (2006). Current validity of the Delphi method in social sciences. Technol. Forecast. Soc. Change 73, 467-482. doi: 10.1016/j.techfore.2005.09.002

MacMillan, D., Hanley, N., and Lienhoop, N. (2006). Contingent valuation: environmental polling or preference engine? Ecol. Econ. 60, 299-307. doi: 10.1016/j.ecolecon.2005.11.031

MEA (2005). Ecosystems and Human Well-Being: Synthesis. Washington, DC: Island Press.

Morelli, F., and Møller, A. P. (2015). Concerns about the use of ecosystem services as a tool for nature conservation: from misleading concepts to providing a "price" for nature, but not a "value". Eur. J. Ecol. 1, 68-70. doi: 10.1515/eje-20150009

MSFD (2008). Marine Strategy Framework Directive. Brussels: European Union.

Nienstedt, K. M., Brock, T. C. M., Van Wensem, J., Montforts, M., Hart, A., Aagaard, A., et al. (2012). Development of a framework based on an ecosystem services approach for deriving specific protection goals for environmental risk assessment of pesticides. Sci. Total Environ. 415(Suppl. C), 31-38. doi: 10.1016/ j.scitotenv.2011.05.057

Ntona, M., and Morgera, E. (2018). Connecting SDG 14 with the other sustainable development goals through marine spatial planning. Mar. Policy 93, 214-222. doi: 10.1016/j.marpol.2017.06.020

Oevelen, D. V., Duineveld, G., Lavaleye, M., Mienis, F., Soetaert, K., and Heip, C. H. R. (2009). The cold-water coral community as hotspot of carbon cycling on continental margins: a food-web analysis from Rockall Bank (northeast Atlantic). Limnol. Oceanogr. 54, 1829-1844. doi: 10.4319/lo.2009.54. 6.1829

Olita, A., Cucco, A., Simeone, S., Ribotti, A., Fazioli, L., Sorgente, B., et al. (2012). Oil spill hazard and risk assessment for the shorelines of a Mediterranean coastal archipelago. Ocean Coast. Manage. 57(Suppl. C), 44-52. doi: 10.1016/ j.ocecoaman.2011.11.006

Ramirez-Llodra, E., Tyler, P. A., Baker, M. C., Bergstad, O. A., Clark, M. R., Escobar, E., et al. (2011). Man and the last great wilderness: human impact on the deep sea. PLoS One 6:e22588. doi: 10.1371/journal.pone. 0022588

Scolozzi, R., Morri, E., and Santolini, R. (2012). Delphi-based change assessment in ecosystem service values to support strategic spatial planning in Italian landscapes. Ecol. Indic. 21, 134-144. doi: 10.1016/j.ecolind.2011.07.019

Silvertown, J. (2015). Have ecosystem services been oversold? Trends Ecol. Evol. 30, 641-648. doi: 10.1016/j.tree.2015.08.007

Soares, C. G., and Teixeira, A. P. (2001). Risk assessment in maritime transportation. Reliab. Eng. Syst. Safety 74, 299-309. doi: 10.1016/S09518320(01)00104- 1

Staples, D., Brainard, R., Capezzuoli, S., Funge-Smith, S., Grose, C., Heenan, A., et al. (2014). Essential EAFM. Ecosystem Approach to Fisheries Management Training Course, Vol. 1. Bangkok: RAP Publication, 13.

Steen, J. T. V. D., Radbruch, L., Hertogh, C. M., Boer, M. E. D., Hughes, J. C., Larkin, P., et al. (2014). White paper defining optimal palliative care in older people with dementia: a Delphi study and recommendations from the European Association for Palliative Care. Palliat. Med. 28, 197-209. doi: 10.1177/0269216313493685

Stenseke, M., and Larigauderie, A. (2018). The role, importance and challenges of social sciences and humanities in the work of the intergovernmental sciencepolicy platform on biodiversity and ecosystem services (IPBES). Innovation 31(Suppl.1), S10-S14. doi: 10.1080/13511610.2017.1398076

Stirling, A. (1998). Risk at a turning point? J. Risk Res. 1, 97-109. doi: 10.1080/ 136698798377204

Strand, J., Carson, R. T., Navrud, S., Ortiz-Bobea, A., and Vincent, J. R. (2017). Using the Delphi method to value protection of the Amazon rainforest. Ecol. Econ. 131(Suppl. C), 475-484. doi: 10.1016/j.ecolecon.2016.09.028

Sullivan, S. (2010). 'Ecosystem service commodities' - A new imperial ecology? Implications for animist immanent ecologies, with deleuze and guattari. New Format. 69, 111-128. doi: 10.3898/NEWF.69.06.2010

Sweetman, A. K., Thurber, A. R., Smith, C. R., Levin, L. A., Mora, C., Wei, C. L., et al. (2017). Major impacts of climate change on deep-sea benthic ecosystems. Elem. Sci. Anth. 5:4. doi: 10.1525/elementa.203

TEEB (2010). The Economics of Ecosystems and Biodiversity. Ecological and Economic Foundations. Washington DC: Earthscan.

Thurber, A. R., Sweetman, A. K., Narayanaswamy, B. E., Jones, D. O. B., Ingels, J., and Hansman, R. L. (2014). Ecosystem function and services provided by the deep sea. Biogeosciences 11, 3941-3963. doi: 10.5194/bg-11-39412014

Toppinen, A., Pätäri, S., Tuppura, A., and Jantunen, A. (2017). The European pulp and paper industry in transition to a bio-economy: a Delphi study. Futures 88(Suppl. C), 1-14. doi: 10.1016/j.futures.2017. 02.002

Vanreusel, A., Fonseca, G., Danovaro, R., Da Silva, M. C., Esteves, A. M., Ferrero, T., et al. (2010). The contribution of deep-sea macrohabitat heterogeneity to global nematode diversity. Mar. Ecol. 31, 6-20. doi: 10.1111/ j.1439-0485.2009.00352.x

Woudenberg, F. (1991). An evaluation of Delphi. Technol. Forecast. Soc. Change 40, 131-150. doi: 10.1016/0040-1625(91)90002-W

Conflict of Interest Statement: The authors declare that the research was conducted in the absence of any commercial or financial relationships that could be construed as a potential conflict of interest.

Copyright (C) 2019 Armstrong, Vondolia, Foley, Henry, Needham and Ressurreição. This is an open-access article distributed under the terms of the Creative Commons Attribution License (CC BY). The use, distribution or reproduction in other forums is permitted, provided the original author(s) and the copyright owner(s) are credited and that the original publication in this journal is cited, in accordance with accepted academic practice. No use, distribution or reproduction is permitted which does not comply with these terms. 